\title{
Design and Analysis of an MST-Based Topology Control Algorithm
}

\author{
Ning Li, Jennifer C. Hou, and Lui Sha \\ Department of Computer Science \\ University of Illinois at Urbana-Champaign \\ Urbana, IL 61801 \\ \{nli, jhou, lrs\}@cs.uiuc.edu
}

\begin{abstract}
In this paper, we present a Minimum Spanning Tree (MST) based topology control algorithm, called Local Minimum Spanning Tree (LMST), for wireless multi-hop networks. In this algorithm, each node builds its local minimum spanning tree independently and only keeps on-tree nodes that are one-hop away as its neighbors in the final topology. We analytically prove several important properties of LMST: (1) the topology derived under LMST preserves the network connectivity; (2) the node degree of any node in the resulting topology is bounded by 6; and (3) the topology can be transformed into one with bi-directional links (without impairing the network connectivity) after removal of all uni-directional links. These results are corroborated in the simulation study.
\end{abstract}

\section{INTRODUCTION}

Topology control and management - how to determine the transmission power of each node so as to maintain network connectivity while consuming the minimum possible power has emerged to be one of the most important issues in wireless multi-hop networks [1]. Instead of transmitting using the maximum possible power, nodes in a wireless multi-hop network collaboratively determine their transmission power and define the topology of the wireless network by the neighbor relation under certain criteria. This is in contrast to the "traditional" network in which each node transmits using its maximum transmission power and the topology is built implicitly by routing protocols (that update their routing caches as timely as possible) [2] [3] without considering the power issue. Not until recently has the issue of topology/power control with respect to maintaining network connectivity, optimizing network spatial reuse, and mitigating MAC-level interference attracted much attention.

The importance of topology control lies in the fact that it critically affects the system performance in several ways. For one, as shown in [4], it affects network spatial reuse and hence the traffic carrying capacity. Choosing too large a power level results in excessive interference, while choosing too small a power level results in a disconnected network. Power control also effects the energy usage of communication, thus impacts on battery life, a critical resource in many mobile applications. In addition, topology control also impacts on contention for the medium. Collisions can be mitigated as much as possible by choosing the smallest transmission power subject to maintaining network connectivity [5] [6].
Several topology control algorithms [5], [7]-[9] have been proposed to create a power-efficient network topology in wireless multi-hop networks with limited mobility. We will summarize the existing work in Section II. Some of the algorithms require explicit propagation channel models (e.g., [9]), while others incur significant message exchanges (e.g., [5]). Their ability to maintain the topology in the case of mobility is also rather limited.

In this paper, we propose a Minimum Spanning Tree (MST) based topology control algorithm, called Local Minimum Spanning Tree (LMST), for multi-hop wireless networks with limited mobility. The topology is constructed by each node building its local MST independently (with the use of information locally collected) and only keeping one-hop on-tree nodes as neighbors. The contributions of this paper include: (i) the topology constructed under LMST preserves the network connectivity, (ii) the degree of any node in the resulting topology is bounded by 6 ; and (iii) the resulting topology can be converted into one with only bi-directional links (after removal of uni-directional links). Feature (ii) is desirable because a small node degree reduces the MAC-level contention and interference. The capability of forming a topology that consists of only bi-directional links is important for link level acknowledgments, and critical for packet transmissions and retransmissions over the unreliable wireless medium. Bidirectional links are also important for the medium access control mechanisms such as RTS/CTS in IEEE 802.11.

The rest of the paper is organized as follows. The related work is firstly summarized in Section II. Then we present the LMST algorithm in Section III, and prove its properties: preservation of network connectivity, bound on the node degree, and construction of topology with only bi-directional links, in Section IV. The frequency to update the topology in case of limited mobility is determined under a probabilistic model in Section IV. Finally, we present a simulation-based performance study in Section $\mathrm{V}$, and conclude the paper in Section VI.

\section{RELATED WORK}

As mentioned in the previous section, several topology control algorithms have been proposed in the literature, among which the relay-region and enclosure-based approach [9], 
$\operatorname{CBTC}(\alpha)$ [7], COMPOW [5], and CONNECT [8] may have received the most attention. Several broadcast/multicast algorithms for ad-hoc wireless networks ( [10] [11] [12] [13] [14]) have also attempted to maintain some type of overlay topology, upon which a multicast tree/mesh can be built. The issue of constructing an overlay topology to facilitate multicast tree/mesh building is outside the scope of this paper.

Relay-region and enclosure-based approach (R\&M): Rodoplu et al. [9] introduced the notion of relay region and enclosure for the purpose of power control. For any node $i$ that intends to transmit to node $j$, node $j$ is said to lie in the relay region of a third node $r$, if node $i$ will consume less power when it chooses to relay through node $r$ instead of transmitting directly to node $j$. The enclosure of node $i$ is then defined as the union of the complement of relay regions of all the nodes that node $i$ can reach by using its maximal transmission power. It is shown that the network is strongly connected if every node maintains links with the nodes in its enclosure and the resulting topology is a minimum power topology.

A two-phase distributed protocol was then devised to find the minimum power topology for a static network. In the first phase, each node $i$ executes local search to find the enclosure graph. This is done by examining neighbor nodes which a node can reach by using its maximal power and keeping only those do not lie in the relay regions of previously found nodes. In the second phase, each node runs the distributed Bellman-Ford shortest path algorithm upon the enclosure graph, using the power consumption as the cost metric. When a node completes the second phase, it can either start data transmission or enter the sleep mode to conserve power.

To deal with limited mobility, each node periodically executes the distributed protocol to find the enclosure graph. This algorithm assumes that there is only one data sink (destination) in the network, which may not hold in practice. Also, an explicit propagation channel model is needed to compute the relay region.

CONNECT and its extension: Ramanathan et al. [8] presented two centralized algorithms to minimize the maximum power used per node while maintaining the (bi)connectivity of the network. CONNECT is a simple greedy algorithm that iteratively merges different components until only one remains. Augmenting a connected network to a bi-connected network is done by BICONN-AUGMENT, which uses the same idea as in CONNECT to iteratively build the bi-connected network. In addition, a post-processing phase can be applied to ensure per-node minimality by deleting redundant connections.

Two distributed heuristics are introduced for mobile networks. In LINT, each node is configured with three parameters - the "desired" node degree $d_{d}$, a high threshold $d_{h}$ on the node degree, and a low threshold $d_{l}$. Every node will periodically check the number of active neighbors and change its power level accordingly, so that the node degree is kept within the thresholds. LILT further improves LINT by overriding the high threshold when the topology change indicated by the routing update results in undesirable connectivity.
Both CONNECT and BICONN-AUGMENT are centralized algorithms that requires global information, thus cannot be directly deployed in the case of mobility. On the other hand, the proposed heuristics LINT and LILT cannot guarantee the preservation of the network connectivity.

COMPOW: Narayanaswamy et al. [5] developed a power control protocol, called COMPOW. The authors argued that if each node uses the smallest common power required to maintain the network connectivity, the traffic carrying capacity of the entire network is maximized, the battery life is extended, and the contention at the MAC layer is reduced. In COMPOW each node runs several routing daemons in parallel, one for each power level. Each routing daemon maintains its own routing table by exchanging control messages at the specified power level. By comparing the entries in different routing tables, each node can determine the smallest common power that ensures the maximal number of nodes are connected. Specifically, let $N\left(P_{i}\right)$ denote the number of entries in the routing table corresponding to the power level $P_{i}$. Then the adequate power level for data packets is simply set to the smallest power level $P_{i}$ for which $N\left(P_{i}\right)=N\left(P_{\max }\right)$.

The major drawback of COMPOW is its significant message overhead, since each node runs multiple daemons, each of which has to exchange link state information with the counterparts at other nodes. COMPOW also tends to use higher power in the case of unevenly distributed nodes. Finally, since the common power is collaboratively determined by the all nodes inside the network, global reconfiguration is required in the case of node joining/leaving.

$\operatorname{CBTC}(\alpha)$ : The work that comes closest to our work is $\operatorname{CBTC}(\alpha)$ [7]. $\operatorname{CBTC}(\alpha)$ is a two-phase algorithm in which each node finds the minimum power $p$ such that transmitting with $p$ ensures that it can reach some node in every cone of degree $\alpha$. The algorithm has been analytically shown to preserve the network connectivity if $\alpha<5 \pi / 6$. It has also ensured that every link between nodes is bi-directional.

Several optimizations to the basic algorithm are also discussed, which include: (i) a shrink-back operation can be added at the end to allow a boundary node to broadcast with less power, if doing so does not reduce the cone coverage; (ii) if $\alpha<2 \pi / 3$, asymmetric edges can be removed while maintaining the network connectivity; and (iii) if there exists an edge from $u$ to $v_{1}$ and from $u$ to $v_{2}$, respectively, the longer edge can be removed while preserving connectivity, as long as $d\left(v_{1}, v_{2}\right)<\max \left\{d\left(u, v_{1}\right), d\left(u, v_{2}\right)\right\}$.

An event-driven strategy is proposed to reconfigure the network topology in the case of mobility. Each node is notified when any neighbor leaves/joins the neighborhood and/or the angle changes. The mechanism used to realize this requires state to be kept at, and message exchanges among, neighboring nodes. The node then determines whether it needs to rerun the topology control algorithm.

Other power-efficient topology control work: There also exists work in generating power-efficient topology in wireless networks. By following a probabilistic approach, Santi et al. derived the suitable common transmission range which 
preserves network connectivity, and established the lower and upper bounds on the probability of connectedness [6]. In [15], a "backbone protocol" is proposed to manage large wireless ad hoc networks, in which a small subset of nodes is selected to construct the backbone. In [16], a method of calculating the power-aware connected dominating sets was proposed to establish an underlying topology for the network.

\section{The MSt-Based Topology Control Algorithm}

In this section, we first outline a set of guidelines for devising topology control algorithms. Then we present a distributed topology control algorithm called LMST (Local Minimum Spanning Tree).

\section{A. Design Guidelines}

The following guidelines are essential to an effective topology control algorithm:

1) The network connectivity should be preserved with the use of minimal possible power. This is the most important objective of topology control algorithms.

2) The algorithm should be distributed. This is due to the fact that there is, in general, no central authority in a wireless multi-hop network, thus each node has to make its decision based on the information it has collected from the network.

3) To be less susceptible to the impact of mobility, the algorithm should depend only on the information collected locally, e.g., information collected within one hop. Algorithms that depend only on local information also incur less message overhead/delay in the process of collecting information.

4) It is desirable that all links are bi-directional. As mentioned in Section I, bi-directional links facilitate linklevel acknowledgment, proper operation of the RTS/CTS mechanism, and ensures existence of reverse paths [5].

5) It is also desirable that the node degree in the topology derived under the algorithm is small. A small node degree may help to mitigate the well known hidden and exposed terminal problems, ${ }^{1}$ as there will not be so many nodes that have to be silenced in a communication activity.

\section{B. The LMST Algorithm}

To facilitate discussion of the proposed algorithm, we first define the following terms. We denote the network topology constructed under the common maximum transmission range $d_{\max }$ as an undirected simple graph $G=(V, E)$ in the plane, where $V$ is the set of nodes in the network and $E=\{(u, v)$ : $\left.d(u, v) \leq d_{\max }, u, v \in V\right\}$ is the edge set of $G$. A unique $i d$ (such as an IP/MAC address) is assigned to each node. For

\footnotetext{
${ }^{1}$ The hidden terminal problem refers to the situation in which a station is hidden when it is within the transmission range of the intended receiver node of the packet but out of the range of the sender node, where the exposed terminal problem refers to the situation in which a station is exposed when it is within the transmission range of the sender node, but out of the range of the receiver.
}

notational simplicity, we denote $i d\left(v_{i}\right)=i$. We also define the Visible Neighborhood $N V_{u}(G)$ of node $u$ as follows.

Definition 1 (Visible Neighborhood): The visible neighborhood $N V_{u}(G)$ is the set of nodes that node $u$ can reach by using the maximum transmission power, i.e., $N V_{u}(G)=$ $\left\{v \in V(G): d(u, v) \leq d_{\max }\right\}$. For each node $u \in V(G)$, let $G_{u}=\left(V_{u}, E_{u}\right)$ be an induced subgraph of $G$ such that $V_{u}=N V_{u}$.

The proposed algorithm is composed of the following three phases: information collection, topology construction, and determination of transmission power, and an optional optimization phase: construction of topology with only bidirectional edges. We assume that the propagation channel is symmetric and obstacle-free, and each node is equipped with the ability to gather its location information via, for example, GPS for outdoor applications and pseudolite [17] for indoor applications.

1) Information Exchange: The information needed by each node $u$ in the topology construction process is the information of all nodes in $N V_{u}(G)$. This can be obtained by having each node broadcast periodically a Hello message using its maximal transmission power. The information contained in a Hello message should at least include the node id and the position of the node. These periodic messages can be sent either in the data channel or in a separate control channel. ${ }^{2}$ The time interval between two broadcasts of Hello messages depends on the level of nodal mobility, and will be determined by the probabilistic model to be introduced in Section IV-B.

2) Topology Construction: After obtaining the information of visible neighborhood $N V_{u}(G)$, each node $u$ applies Prim's Algorithm [18] independently to obtain its local minimum spanning tree $T_{u}=\left(V\left(T_{u}\right), E\left(T_{u}\right)\right)$ of $G_{u}$. Note that the time complexity of Prim's algorithm is $O(n \log n+e \log n)=$ $O(e \log n)$, where $n$ is the number of nodes and $e$ is the number of edges in $G_{u}$. This can be improved using Fibonacci Heaps to $O(e+\log n)$ [19].

Two points are worth mentioning here. Firstly, to build a power efficient minimum spanning tree, the weight of an edge should be the transmission power between the two nodes. As power consumption is, in general, of the form $c \cdot d^{r}, r \geq 2$, i.e., a strictly increasing function of the Euclidean distance, it suffices to use the Euclidean distance as the weight function. The same minimum spanning tree will result. Secondly, the minimum spanning tree derived under Prim's algorithm may not be unique if there exist multiple edges with the same weight. The uniqueness is necessary for the proof of connectivity, thus we refine the weight function as follows:

Definition 2 (Weight Function): Given two edges $\left(u_{1}, v_{1}\right)$ and $\left(u_{2}, v_{2}\right)$, the weight function $d^{\prime}: E \mapsto R$ is defined as:

$$
\begin{aligned}
& d^{\prime}\left(u_{1}, v_{1}\right)>d^{\prime}\left(u_{2}, v_{2}\right) \\
& \quad \Leftrightarrow \quad d\left(u_{1}, v_{1}\right)>d\left(u_{2}, v_{2}\right) \\
& \quad \text { or } \quad\left(d\left(u_{1}, v_{1}\right)=d\left(u_{2}, v_{2}\right)\right.
\end{aligned}
$$

${ }^{2}$ Each node can piggyback its location information in data packets to reduce the number of Hello exchanges. 


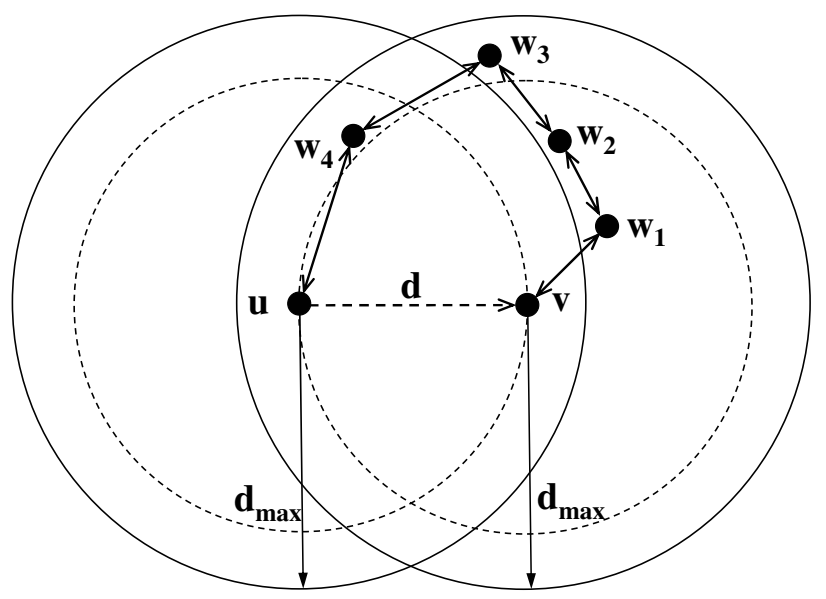

Fig. 1. A scenario that demonstrates that links in the topology derived under LMST may be uni-directional.

$$
\begin{aligned}
\left.\& \& \max \left\{i d\left(u_{1}\right), i d\left(v_{1}\right)\right\}>\max \left\{i d\left(u_{2}\right), i d\left(v_{2}\right)\right\}\right) \\
\text { or } \quad\left(d\left(u_{1}, v_{1}\right)=d\left(u_{2}, v_{2}\right)\right. \\
\& \& \max \left\{i d\left(u_{1}\right), i d\left(v_{1}\right)\right\}=\max \left\{i d\left(u_{2}\right), i d\left(v_{2}\right)\right\} \\
\left.\& \& \min \left\{i d\left(u_{1}\right), i d\left(v_{1}\right)\right\}>\min \left\{i d\left(u_{2}\right), i d\left(v_{2}\right)\right\}\right) .
\end{aligned}
$$

The weight function $d^{\prime}$ guarantees that in each step of Prim's algorithm, the choice on the minimum weight edges $e$ is unique, thus the local minimum spanning tree $T_{u}$ constructed by node $u$ is unique.

After node $u$ builds a minimum spanning tree to span its visible neighborhood, it will determine its neighbors. To facilitate discussion, we define the Neighbor Relation and the Neighbor Set:

Definition 3 (Neighbor Relation and Neighbor Set): Node $v$ is a neighbor of node $u$ 's, denoted $u \rightarrow v$, if and only if $(u, v) \in E\left(T_{u}\right) . u \leftrightarrow v$ if and only if $u \rightarrow v$ and $v \rightarrow u$. That is, node $v$ is a neighbor of node $u$ 's if and only if node $v$ is on node $u$ 's minimum spanning tree, $T_{u}$, and is "one-hop" away from node $u$. The neighbor set $N(u)$ of node $u$ is $N(u)=\left\{v \in V\left(G_{u}\right): u \rightarrow v\right\}$.

The neighbor relation defined above is not symmetric, i.e., $u \rightarrow v$ does not necessarily imply $v \rightarrow u$. Figure 1 gives such an example. There are altogether 6 nodes, $V=\left\{u, v, w_{1}, w_{2}, w_{3}, w_{4}\right\}$, where $d(u, v)=d<d_{\max }$, $d\left(u, w_{4}\right)<d_{\max }, d\left(u, w_{i}\right)>d_{\max }, i=1,2,3$, and $d\left(v, w_{j}\right)<d_{\max }, j=1,2,3,4$. Since $N V_{u}=\left\{u, v, w_{4}\right\}$, it can be obtained from $T_{u}$ that $u \rightarrow v$ and $u \rightarrow w_{4}$. Also $V N_{v}=\left\{u, v, w_{1}, w_{2}, w_{3}, w_{4}\right\}$, thus $v \rightarrow w_{1}$. Here we have $u \rightarrow v$ but $v \nrightarrow u$.

The network topology under LMST is all the nodes in $V$ and their individually perceived neighbor relations (note that it is not a simple superposition of all local MSTs).

Definition 4 (Topology $G_{0}$ ): The topology, $G_{0}$, derived under LMST is a directed graph $G_{0}=\left(V_{0}, E_{0}\right)$, where $V_{0}=V$, $E_{0}=\{(u, v): u \rightarrow v, u, v \in V(G)\}$.

3) Determination of Transmission Power: Assume that the maximal transmission power is known and is the same to all nodes. By measuring the receiving power of Hello messages, each node can determine the specific power levels it needs to reach each of its neighbors. This approach can be applied to any propagation channel model. In what follows, we first describe two commonly-used propagation models, and then elaborate on how we determine the transmission power.

In the Free Space propagation model, the relation between the power used to transmit packets, $P_{t}$ and the power received, $P_{r}$ can be characterized as

$$
P_{r}=\frac{P_{t} G_{t} G_{r} \lambda^{2}}{(4 \pi d)^{2} L}
$$

where $G_{t}$ is the antenna gain of the transmitter, $G_{r}$ is the antenna gain of the receiver, $\lambda$ is the wave length, $d$ is the distance between the antenna of the transmitter and that of the receiver, and $L$ is the system loss.

In the Two-Ray Ground propagation model, the relation between $P_{t}$ and $P_{r}$ is

$$
P_{r}=\frac{P_{t} G_{t} G_{r} h_{t}^{2} h_{r}^{2}}{d^{4} L},
$$

where $G_{t}$ is the antenna gain of the transmitter, $G_{r}$ is the antenna gain of the receiver, $h_{t}$ is the antenna height of the transmitter, $h_{r}$ is the antenna height of the receiver, $d$ is the distance between the antenna of the transmitter and that of the receiver, and $L$ is the system loss.

In general, the relation between $P_{t}$ and $P_{r}$ is of the following form

$$
P_{r}=P_{t} \cdot G,
$$

where $G$ is a function of $G_{t}, G_{r}, h_{t}, h_{r}, \lambda, d, \alpha, L$ and is timeinvariant if all the above parameters are time-invariant. At the information exchange stage, each node broadcasts its position using the maximal transmission power $P_{\max }$. When node $A$ receives the position information from node $B$, it measures the receiving power $P_{r}$ and obtains $G$

$$
G=P_{r} / P_{\max } .
$$

Henceforth node $A$ needs to transmit using at least $P_{t h} \cdot G=$ $P_{t h} P_{r} / P_{\max }$ so that node $B$ can receive messages, where $P_{t h}$ is the power threshold to correctly understand the message.

A broadcast to all neighbors requires a power level that can reach the farthest neighbor. Here we introduce the notion of Radius:

Definition 5 (Radius of Node $u$ ): The radius, $r_{u}$, of node $u$ is defined as the distance between node $u$ and its farthest neighbor (in terms of Euclidean distance), i.e, $r_{u}=$ $\max \{d(u, v): v \in N(u)\}$.

4) Construction of Topology with Only Bi-Directional Edges: As illustrated in Figure 1, some links in $G_{0}$ may be uni-directional. As mentioned in Section III-A, it is desirable to obtain network topologies consisting of only bi-directional edges. There are two possible solutions: (i) to enforce all the uni-directional links in $G_{0}$ to become bi-directional; or (ii) to delete all the uni-directional links in $G_{0}$. We term the two new topologies $G_{0}^{+}$and $G_{0}^{-}$, respectively. Specifically, 


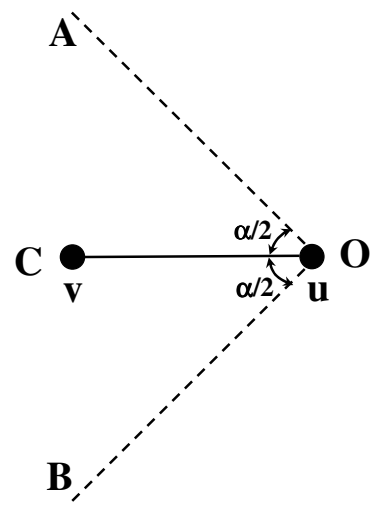

Fig. 2. The definition of cone $(u, \alpha, v)$.

Definition 6 (Topology $G_{0}^{+}$): The topology, $G_{0}^{+}$, is a undirected graph $G_{0}^{+}=\left(V_{0}^{+}, E_{0}^{+}\right)$, where $V_{0}^{+}=V_{0}, E_{0}^{+}=$ $\left\{(u, v):(u, v) \in E\left(G_{0}\right)\right.$ or $\left.(v, u) \in E\left(G_{0}\right)\right\}$.

Definition 7 (Topology $G_{0}^{-}$): The topology, $G_{0}^{-}$, is a undirected graph $G_{0}^{-}=\left(V_{0}^{-}, E_{0}^{-}\right)$, where $V_{0}^{-}=V_{0}, E_{0}^{-}=$ $\left\{(u, v):(u, v) \in E\left(G_{0}\right)\right.$ and $\left.(v, u) \in E\left(G_{0}\right)\right\}$.

To convert $G_{0}$ into either $G_{0}^{+}$or $G_{0}^{-}$, every node $u$ may probe each of its neighbors in the neighbor set $N(u)$ to find out whether or not the corresponding edge is uni-directional, and in the case of a uni-directional edge, either deletes the edge $\left(G_{0}^{-}\right)$or notifies its neighbor to add the reverse edge $\left(G_{0}^{+}\right)$. In Section IV, we will prove that both new topologies preserve the desirable properties of $G_{0}$. There exists a tradeoff between the two choices: the latter gives a comparatively simpler topology, and hence is more efficient in terms of spatial reuse, while the former keeps more routing redundancy.

\section{Theoretical Base of LMST}

In this section, we state and prove several desirable properties of the network topology derived by LMST. We also determine, with the use of a probabilistic model, how often the neighborhood information should be exchanged and the topology should be updated.

\section{A. Properties of LMST}

Definition 8 (Cone): As shown in Figure 2, a cone $(u, \alpha, v)$ is the region in the plane that lies between $\overline{O A}$ and $\overline{O B}$, where $\angle C O A=\angle C O B=\alpha / 2$.

1) Degree Bound: It has been observed that any minimum spanning tree of a finite set of points in the plane has a maximum node degree of six [20]. We prove this property (which will serve as the base for the proof of Theorem 3) independently in the context of topology control.

Lemma 1: Given three nodes $u, v, w \in V\left(G_{0}\right)$ satisfying $d^{\prime}(u, v)>d^{\prime}(u, w)$ and $d^{\prime}(u, v)>d^{\prime}(v, w)$, then $u \nrightarrow v$.

Proof: If $d(u, v)>d_{\max }$, then $u \nrightarrow v$. Thus, we only need to consider the case $d(u, v) \leq d_{\max }$. Assume $u \rightarrow v$. Then $(u, v) \in E\left(T_{u}\right)$. It follows that only one of the two edges $(u, w)$ and $(v, w)$ can be in $E\left(T_{u}\right)$ (otherwise a loop is formed). Without loss of generality, assume $(u, w) \in E\left(T_{u}\right)$. Since $(v, w) \notin E\left(T_{u}\right)$ and $d^{\prime}(v, w)<d^{\prime}(u, v)$, replacing

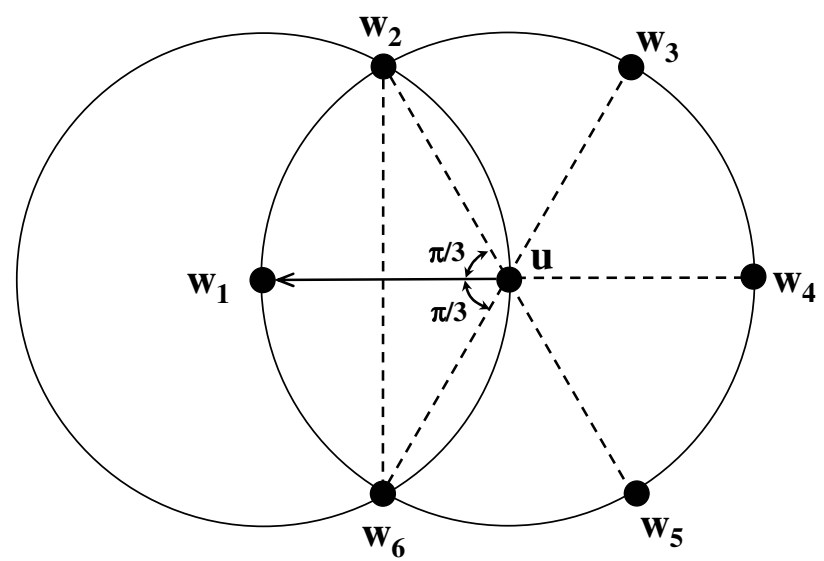

Fig. 3. The degree of any node in $G_{0}$ is bounded by 6 .

edge $(u, v)$ with edge $(v, w)$ and keeping all other edges unchanged in $E\left(T_{u}\right)$ results in another spanning tree of $G_{u}$ with a less weight. This contradicts the fact that $T_{u}$ is the unique minimum spanning tree of $G_{u}$.

Theorem 1 (Degree Bound): Define the degree of a node as the number of neighbors. The degree of any node in $G_{0}$ is bounded by 6 , i.e., $\operatorname{deg}(u) \leq 6, \forall u \in V\left(G_{0}\right)$.

Proof: Consider any node $u \in V\left(G_{0}\right)$. Order nodes in $N(u)$ by their distances from $u$, such that for the $i$ th node $w_{i}$ and the $j t h$ node $w_{j}, j>i$, we have $d\left(u, w_{j}\right) \geq d\left(u, w_{i}\right)$. By Lemma 1 , we have $d\left(u, w_{j}\right) \leq d\left(w_{i}, w_{j}\right)$, otherwise $u \nrightarrow w_{j}$. Thus node $w_{j}$ cannot reside inside Cone $\left(u, 2 \pi / 3, w_{i}\right)$. That is, as shown in Figure 3, node $u$ cannot have neighbors other than node $w_{1}$ inside Cone $\left(u, 2 \pi / 3, w_{1}\right)$. By induction on the rank of nodes in $N(u)$, the maximal number of neighbors that $u$ can have is no greater than 6 , i.e., $\operatorname{deg}(u) \leq 6$. Note that Figure 3 also depicts the only scenario in which $\operatorname{deg}(u)=6$ occurs.

In wireless multi-hop networks, one observation is that less node degree usually results in less contention and interference. The degree bound obtained in Theorem 1 can be very important to scheduling algorithms. As matter of fact, several TDMA-based scheduling algorithms have been proposed to maximize the spatial reuse and minimize frame length [21] [22], most of which require that the maximum degree must be bounded.

2) Network Connectivity: We prove that the topology, $G_{0}$, derived under LMST preserves the network connectivity of $G$. For any two nodes $u, v \in V\left(G_{0}\right)$, node $u$ is said to be connected to node $v$ (denoted $u \Leftrightarrow v$ ) if there exists a path $\left(w_{0}=u, w_{1}, \ldots, w_{m-1}, w_{m}=v\right)$ such that $w_{j} \leftrightarrow w_{j+1}, j=$ $0,1, \cdots, m-1$, where $w_{k} \in V\left(G_{0}\right), k=0,1, \cdots, m$. It follows that $u \Leftrightarrow w$ if $u \Leftrightarrow v$ and $v \Leftrightarrow w$.

Lemma 2: For any node pair $[u, v], u, v \in V\left(G_{0}\right)$, if $d(u, v) \leq d_{\max }$ then $u \Leftrightarrow v$.

Proof: For all the node pairs $[u, v]$ satisfying $d(u, v) \leq$ $d_{\max }$ and $u, v \in V\left(G_{0}\right)$, sort them in the increasing order of $d^{\prime}(u, v)$, i.e., $d^{\prime}\left(u_{1}, v_{1}\right)<d^{\prime}\left(u_{2}, v_{2}\right)<\cdots<d^{\prime}\left(u_{l}, v_{l}\right)$. We prove by induction on the rank of the node pairs in the 
ordering.

1) Basis: $k=1$, the first pair $\left[u_{1}, v_{1}\right]$ satisfies $d^{\prime}\left(u_{1}, v_{1}\right)$ $\min _{u, v \in V\left(G_{0}\right)}\left\{d^{\prime}(u, v)\right\}$ and $d\left(u_{1}, v_{1}\right) \leq d_{\max }$. Thı $u \leftrightarrow v$, which means $u \Leftrightarrow v$.

2) Induction: Assume Lemma 2 holds for all pai $\left[u_{i}, v_{i}\right], i=1,2, \cdots, k-1$. Now we prove Lemma also holds for the node pair $\left[u_{k}, v_{k}\right]$. We consider th cases:

- Case 1: $u_{k} \leftrightarrow v_{k}$, which implies $u_{k} \Leftrightarrow v_{k}$.

- Case 2: Either $u_{k} \nrightarrow v_{k}$ or $v_{k} \nrightarrow u_{k}$, or bot Assume $u_{k} \nrightarrow v_{k}$, without loss of generalit Since $v_{k} \in N V_{u_{k}}$, there exists a unique pa $p=\left(w_{0}=u_{k}, w_{1}, w_{2}, \cdots, w_{m-1}, w_{m}=v_{i}\right.$ from node $u_{k}$ to node $v_{k}$, where $\left(w_{i}, w_{i+1}\right)$ $E\left(T_{u_{k}}\right), i=0,1, \cdots, m-1$. Since $T_{u_{k}}$ is tl unique minimum spanning tree of $G_{u_{k}}$, we har $d^{\prime}\left(w_{i}, w_{i+1}\right)<d^{\prime}\left(u_{k}, v_{k}\right)$; otherwise we can col struct another spanning tree with a less weight, $\mathrm{t}$ replacing edge $\left(w_{i}, w_{i+1}\right)$ with $\left(u_{k}, v_{k}\right)$ and keepir all the other edges in $T_{u_{k}}$ unchanged. Applying tl induction hypothesis to each pair $\left[w_{i}, w_{i+1}\right], i$ $0,1, \cdots, m-1$, we have $w_{i} \Leftrightarrow w_{i+1}$, thus $u_{k} \Leftrightarrow v$

Theorem 2: $G_{0}$ preserves the connectivity of $G$, i.e., $G_{0}$ is connected if $G$ is connected.

Proof: Suppose $G$ is connected. We prove by contradiction that $G_{0}$ derived under LMST is a strongly connected graph. Assume $G_{0}$ is not strongly connected. Among all the node pairs $[u, v]$ satisfying $u \nLeftarrow v$, there exists a node pair with the minimum distance, i.e., we can find $\left[v_{0}, v_{1}\right]$ such that $d^{\prime}\left(v_{0}, v_{1}\right)=\min _{u, v \in V\left(G_{0}\right)}\left\{d^{\prime}(u, v): u \nRightarrow v\right\}$. Since $G$ is connected, $d\left(v_{0}, v_{1}\right) \leq d_{\max }$. By Lemma $2, v_{0} \Leftrightarrow v_{1}$, which leads to the contradiction.

3) $G_{0}^{+}$and $G_{0}^{-}$Preserve Properties of $G_{0}: G_{0}^{+}$is an undirected graph, thus all the edges are bi-directional. Since all the links in $G_{0}$ are preserved in $G_{0}^{+}$, it follows that $G_{0}^{+}$ preserves the connectivity of $G_{0}$. Now we prove that the degree of any node in $G_{0}^{+}$is also bounded by 6 . Notice that this is not a simple property of the MST because $G_{0}^{+}$may not be an MST due to those edges added.

Theorem 3: The degree of any node in $G_{0}^{+}$is bounded by 6, i.e., $\operatorname{deg}(u) \leq 6, \forall u \in V\left(G_{0}^{+}\right)$.

Proof: For any node $u \in V\left(G_{0}^{+}\right)$, denote $N^{+}(u)=$ $\left\{(u, v) \in E\left(G_{0}^{+}\right)\right\}$. We prove by contradiction that if $v \in$ $N^{+}(u)$ in $G_{0}^{+}$, there does not exist any other node $w \in N^{+}(u)$ that lies inside Cone $(u, 2 \pi / 3, v)$. Assume that such a node $w \in N^{+}(u)$ exists. We consider four cases:

- Case 1: $u \rightarrow v, u \rightarrow w$ in $G_{0}$. This is proved in Theorem 1 .

- Case 2: $u \rightarrow v, u \nrightarrow w$, but $w \rightarrow u$ in $G_{0}$. We have $d^{\prime}(u, w)>d^{\prime}(u, v)$; otherwise $d^{\prime}(u, v)>d^{\prime}(v, w)$ and $d^{\prime}(u, v)>d^{\prime}(u, w)$, which implies $u \nrightarrow v$ by Lemma 1 . Thus, $d^{\prime}(u, w)>d^{\prime}(v, w)$ and $d^{\prime}(u, w)>d^{\prime}(u, v)$, which implies $w \nrightarrow v$ by Lemma 1. This contradicts the assumption that $w \rightarrow u$.

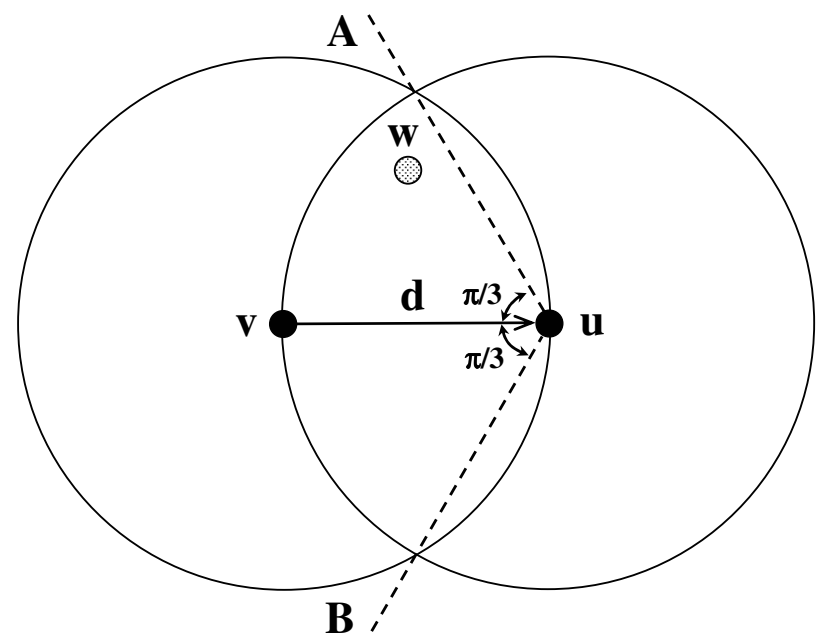

Fig. 4. Proof of Theorem 3.

- Case 3: $u \rightarrow w, u \nrightarrow v$ but $v \rightarrow u$ in $G_{0}$. As shown in Figure 4, we have $d^{\prime}(u, w)<d^{\prime}(u, v)$; otherwise $d^{\prime}(u, w)>d^{\prime}(v, w)$ and $d^{\prime}(u, w)>d^{\prime}(u, v)$, which implies $u \nrightarrow w$ by Lemma 1. Also, we have $d^{\prime}(v, w)>$ $d^{\prime}(v, u)$; otherwise $v \nrightarrow u$ by Lemma 1 . Thus, $d^{\prime}(v, w)>$ $d^{\prime}(u, v)>d^{\prime}(u, w)$, which implies $\angle w u v \geq 2 \pi / 3$. This contradicts the assumption that $w \in \operatorname{Cone}(u, 2 \pi / 3, v)$.

- Case 4: $u \nrightarrow v$ but $v \rightarrow u$, and $u \nrightarrow w$ but $w \rightarrow u$ in $G_{0}$. As shown in Figure 4, we have $d^{\prime}(u, w)>d^{\prime}(u, v)$; otherwise $d^{\prime}(u, v)>d^{\prime}(v, w)$ and $d^{\prime}(u, v)>d^{\prime}(u, w)$, which implies $u \nrightarrow v$ by Lemma 1. Thus $d^{\prime}(u, w)>$ $d^{\prime}(v, w)$ and $d^{\prime}(u, w)>d^{\prime}(u, v)$, which implies $w \nrightarrow v$ by Lemma 1 .

Now we have proved that there does not exist any neighbor other than $v$ that lies inside $\operatorname{Cone}(u, 2 \pi / 3, v)$ in $G_{0}^{+}$. Using the same arguments as in Theorem 1, it is easy to see that the maximal number of neighbors that $u$ can have is no greater than 6, i.e., $\operatorname{deg}(u) \leq 6$.

Since $G_{0}^{-}$is derived from $G_{0}$ by deleting uni-directional links, it is easy to see that the degree of any node in $G_{0}^{-}$ is also bounded by 6 . We now prove that $G_{0}^{-}$preserves the connectivity of $G$.

Theorem 4: $G_{0}^{-}$preserves the connectivity of $G$, i.e., $G_{0}^{-}$ is connected if $G$ is connected.

Proof: If a node pair $[u, v], u, v \in V\left(G_{0}\right)$ satisfies $d(u, v) \leq d_{\max }$, by Lemma 2 , there exists a path $p=\left(w_{0}=\right.$ $\left.u, w_{1}, w_{2}, \ldots, w_{m-1}, w_{m}=v\right)$ such that $w_{j} \leftrightarrow w_{j+1}, j=$ $0,1, \cdots, m-1$, where $w_{k} \in V\left(G_{0}\right), k=0,1, \cdots, m$. The same result holds for $G_{0}^{-}$since all links in $p$ are bi-directional and the removal of uni-directional links does not affect the existence of such a path. Following the same line of argument as presented in Theorem 2, one can prove that $G_{0}^{-}$preserves the connectivity of $G$.

\section{B. Determination of Information Exchange Period}

We determine the time interval between two information exchanges (i.e., two broadcasts of Hello messages) by a probabilistic model with the following assumptions: 


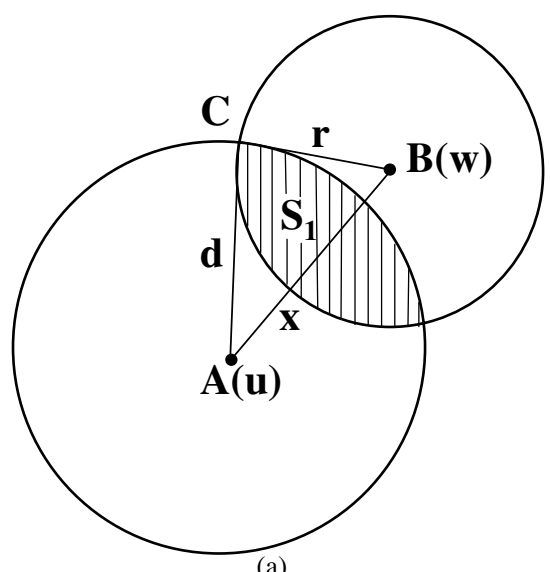

(a)

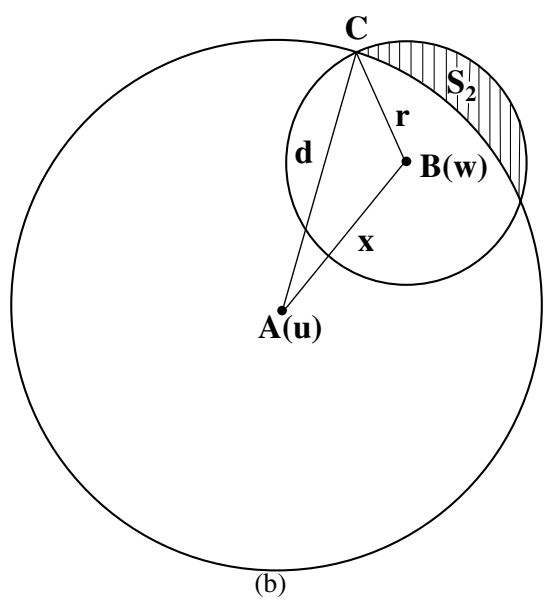

Fig. 5. Calculation of the probabilities that a new neighbor moves into the transmission range of a node and that an existing neighbor moves out of the transmission range, within a time interval of $t$.

(i) All nodes are randomly distributed within a disk of area $S_{0}$ and the total number of nodes in $G, N$, is known.

(ii) For a short time interval of length $t$, each node moves independently toward a random direction in $(0,2 \pi]$, with a constant speed $v$ that is uniformly distributed in $\left[0, v_{\max }\right]$.

(iii) The maximum transmission range of a node is $d=$ $d_{\max }$.

Under these assumptions, one can calculate the probabilities that a new neighbor moves into the transmission range of node $u$ and that an existing neighbor moves out of the transmission range of node $u$, within a time interval of $t$. Here we denote $D(u, d)$ as the disk of radius $d$ centered at node $u$,

1) Probability That Node $w$ Moves Into the Disk $D(u, d)$ : As shown in Figure 5(a), suppose node $u$ is located in position $A$, with its neighbor $w$ in position $B$. The maximum transmission range of node $u$ is $A C=d$, and the distance between nodes $u$ and $w$ is $x(>d)$. Let $B C=r=v_{\max } \cdot t$. The probability that node $w$ moves into the transmission range of node $u$ within time $t$ is the probability that node $w$ moves into the disk $D(u, d)$ (i.e., the shaded area in Figure 5(a)) within time $t$. This probability can be calculated by considering the following two cases:

- Case I: $0<r<2 d$. The probability, $p_{\text {join }}$, that node $w$ moves into $D(u, d)$ within time $t$ is

$$
p_{\text {join }}=\int_{d}^{d+r} \frac{2 \pi x}{S_{0}} \frac{S_{1}}{\pi r^{2}} d x=\int_{d}^{d+r} \frac{2 x S_{1}}{S_{0} r^{2}} d x,
$$

where

$$
\begin{aligned}
& S_{1}=\alpha_{1} d^{2}+\alpha_{2} r^{2}-x r \sin \alpha_{2}, \\
& \alpha_{1}=\angle C A B=\arccos \frac{x^{2}+d^{2}-r^{2}}{2 x d}, \\
& \alpha_{2}=\angle C B A=\arccos \frac{x^{2}+r^{2}-d^{2}}{2 x r} .
\end{aligned}
$$

- Case II: $r \geq 2 d$. The probability of interest is

$$
\begin{aligned}
p_{\text {join }} & =\int_{d}^{r-d} \frac{2 \pi x}{S_{0}} \frac{\pi d^{2}}{\pi r^{2}} d x+\int_{d}^{d+r} \frac{2 \pi x}{S_{0}} \frac{S_{1}}{\pi r^{2}} d x \\
& =\int_{d}^{r-d} \frac{2 \pi x}{S_{0}} \frac{d^{2}}{r^{2}} d x+\int_{r-d}^{r+d} \frac{2 x S_{1}}{S_{0} r^{2}} d x \\
& =\frac{\pi d^{2}}{S_{0} r^{2}}\left[(r-d)^{2}-d^{2}\right]+\int_{r-d}^{r+d} \frac{2 x S_{1}}{S_{0} r^{2}} d x \\
& =\frac{\pi d^{2}(r-2 d)}{S_{0} r}+\int_{r-d}^{r+d} \frac{2 x S_{1}}{S_{0} r^{2}} d x .
\end{aligned}
$$

2) Probability That Node $w$ Moves Out of the Disk $D(u, d)$ : The probability that an existing neighbor $w$ moves out of the maximum transmission range of node $u$ within time $t$ is the probability that $w$ moves out of the disk $D(u, d)$ (i.e., into the shaded area in Figure $5(\mathrm{~b}))$ in time $t$. We consider three cases:

- Case I: $0<r<d$. The probability, $p_{\text {leave }}$, that node $w$ moves out of $D(u, d)$ in time $t$ is

$$
p_{\text {leave }}=\int_{d-r}^{d} \frac{2 \pi x}{S_{0}} \frac{S_{2}}{\pi r^{2}} d x=\int_{d-r}^{d} \frac{2 x S_{2}}{S_{0} r^{2}} d x,
$$

where

$$
\begin{aligned}
S_{2} & =\left(\pi-\alpha_{2}\right) r^{2}-\left(\alpha_{1} d^{2}-x r \sin \alpha_{2}\right), \\
\alpha_{1} & =\angle C A B=\arccos \frac{x^{2}+d^{2}-r^{2}}{2 x d}, \\
\alpha_{2} & =\angle C B A=\arccos \frac{x^{2}+r^{2}-d^{2}}{2 x r} .
\end{aligned}
$$

- Case II: $d \leq r<2 d$. The probability of interest can be expressed as

$$
\begin{aligned}
p_{\text {leave }} & =\int_{0}^{r-d} \frac{2 \pi x}{S_{0}} \frac{\pi\left(r^{2}-d^{2}\right)}{\pi r^{2}} d x+\int_{r-d}^{d} \frac{2 \pi x}{S_{0}} \frac{S_{2}}{\pi r^{2}} d x \\
& =\int_{0}^{r-d} \frac{2 \pi x}{S_{0}} \frac{\left(r^{2}-d^{2}\right)}{r^{2}} d x+\int_{r-d}^{d} \frac{2 x S_{2}}{S_{0} r^{2}} d x
\end{aligned}
$$




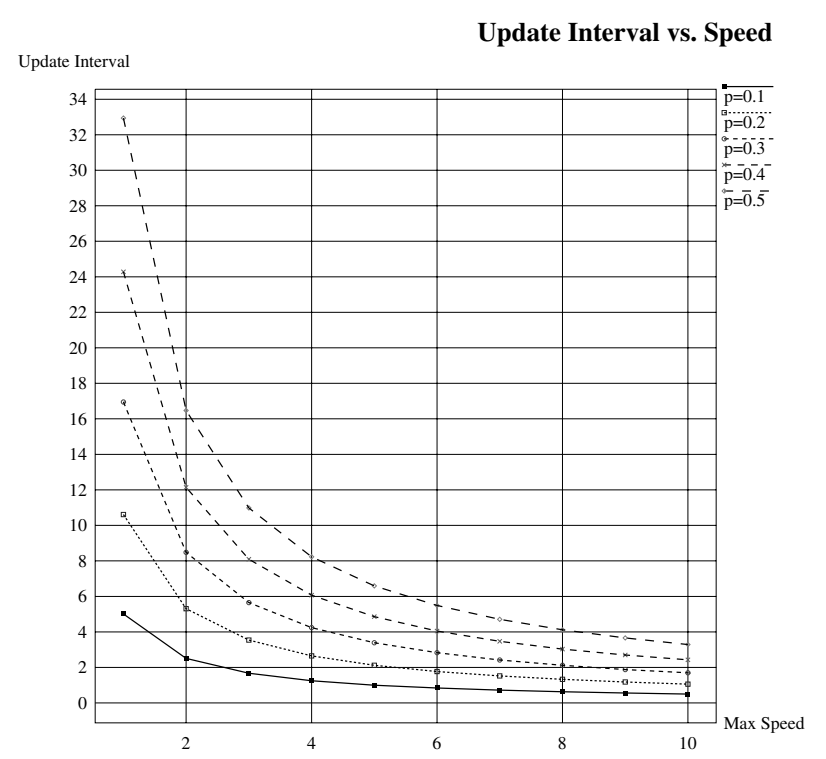

Fig. 6. The information update period versus the maximum speed with respect to different values of $p_{t h}$.

$$
=\frac{\pi(r+d)}{S_{0} r^{2}}(r-d)^{3}+\int_{r-d}^{d} \frac{2 x S_{2}}{S_{0} r^{2}} d x .
$$

- Case III: $r \geq 2 d$. The probability of interest can be expressed as

$$
p_{\text {leave }}=\int_{0}^{d} \frac{2 \pi x}{S_{0}} \frac{\pi\left(r^{2}-d^{2}\right)}{\pi r^{2}} d x=\frac{\pi\left(r^{2}-d^{2}\right) d^{2}}{S_{0} r^{2}} .
$$

3) Determination of information exchange periods: Given that node $u$ has $n$ neighbors and the total number of nodes is $N$. the probability that no new neighbor enters the visible neighborhood of node $u$ is

$$
p_{1}=\left(1-p_{\text {join }}\right)^{N-n-1},
$$

and the probability that no neighbor leaves the visible neighborhood of node $u$ is

$$
p_{2}=\left(1-p_{\text {leave }}\right)^{n} .
$$

Thus, the probability that the visible neighborhood of node $u$ changes is

$$
p_{\text {change }}=1-p_{1} p_{2} .
$$

Given a predetermined probability threshold $p_{t h}$, we can determine the topology update interval $t$ such that $p_{\text {change }}<p_{t h}$.

To demonstrate how the period of information exchange is affected by the maximum speed $v_{\max }$ and the probability threshold $p_{t h}$, we consider a scenario in which 100 nodes are randomly distributed inside a disk of radius $1000 \mathrm{~m}$. The maximum transmission range is $d_{\max }=250 \mathrm{~m}$. The number of neighbors is set to 25. Figure 6 gives the curve of the information update period versus the maximum speed with respect to different values of $p_{t h}$. For example, to ensure the probability of visual neighborhood change is below 0.2 , the information update period decreases from $10.6 \mathrm{sec}$ to 1.06 sec when the maximum nodal speed increases from $1 \mathrm{~m} / \mathrm{s}$ to $10 \mathrm{~m} / \mathrm{s}$.

\section{Performance EVAluation}

In this section, we present several simulation results to demonstrate the effectiveness of LMST. Although network throughput is an important performance metric, it is highly influenced by many other factors, such as MAC protocol, routing protocol, etc. That's the reason why most related work described in Section II doesn't give throughput performance. Instead, we use the following metrics in this paper:

1) Node degree: A smaller average node degree usually implies less contention/interference and better spatial reuse.

2) Radius: As each node $u$ sets $r_{u}$ as its transmission range for broadcasting, a smaller value of the radius implies better network spatial reuse.

3) Average link length: The link length reflects the power level to be used for point-to-point communication. A smaller value of the average link length implies a less power level.

In the simulation study, we compare LMST against R\&M and $C B T C(5 \pi / 6)$ with respect to the above three metrics. Also, the topology generated using the maximum transmission power is used as a baseline. The reason for selecting R\&M and CBTC for comparison is that LMST comes closest to R\&M and CBTC. In particular, (a) CONNECT and its extension are centralized algorithms that require global information, while LMST is a decentralized algorithm that builds the network topology based on information locally gathered; and (b) $C O M P O W$ is known to give poor performance in the case of uneven spatial distributions (as all the nodes have to adopt the transmission power between two most distant neighbors), while the performance of LMST is not subject to the spatial distribution (and as a matter of fact, is especially well-suited in the case of unevenly spatial distributions).

In the first simulation, 100 nodes are uniformly distributed in a $1000 m \times 1000 m$ region. The maximal transmission range is $d_{\max }=250 \mathrm{~m}$ for all the nodes. The topology derived using the maximum transmission power, R\&M (Two-ray ground model), CBTC, LMST, and LMST with link removal are shown in Figure 7(a), (b), (c), and (d), respectively. The corresponding maximum, minimum, and average node degrees in these network topologies are given in Table I. R\&M, CBTC and LMST all dramatically reduce the average node degree, while maintaining network connectivity. Moreover, LMST outperforms both R\&M and CBTC.

In the second simulation, we vary the number of nodes in the region from 50 to 250 . The average node degree for the topologies generated using the maximum transmission power, R\&M, CBTC, and LMST is shown in Figure 8(a), where each data point is an average of 100 simulation runs. The average node degree derived under both $\mathrm{R} \& \mathrm{M}$ and CBTC increases slightly, while the average node degree by LMST does not increase with the spatial density. The average node degree for topologies derived under LMST, LMST with all unidirectional links converted to bi-directional links, and LMST with all uni-directional links removed is shown in Figure 8(b). 


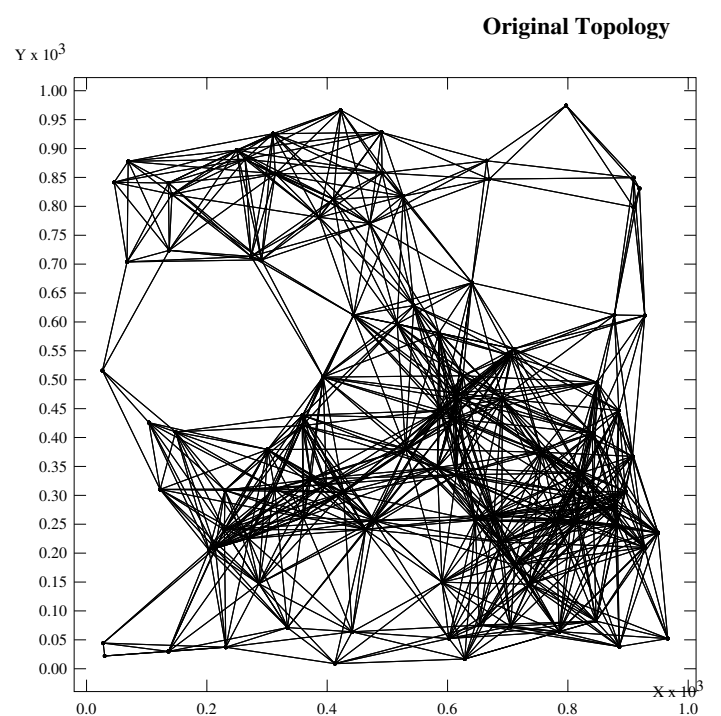

(a) The topology derived using maximum transmission power.

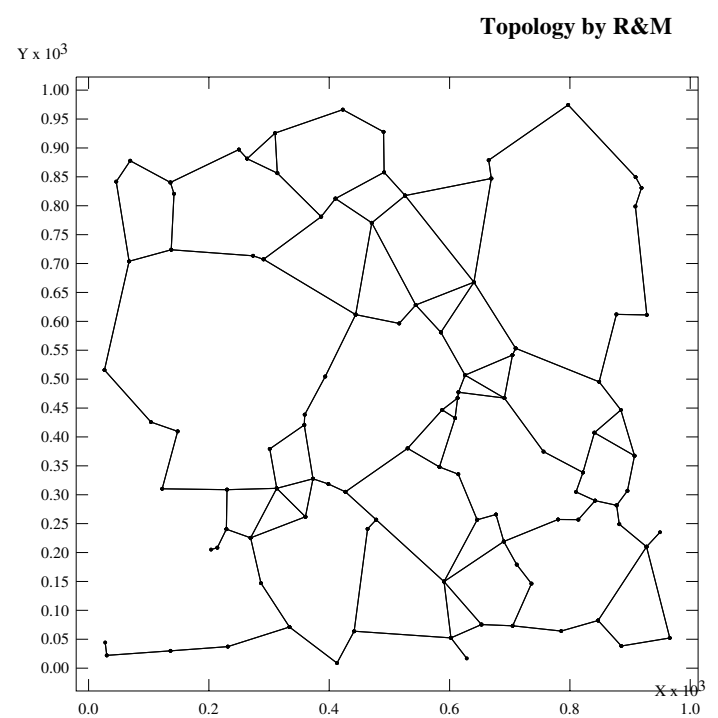

(c) The topology derived under R\&M.

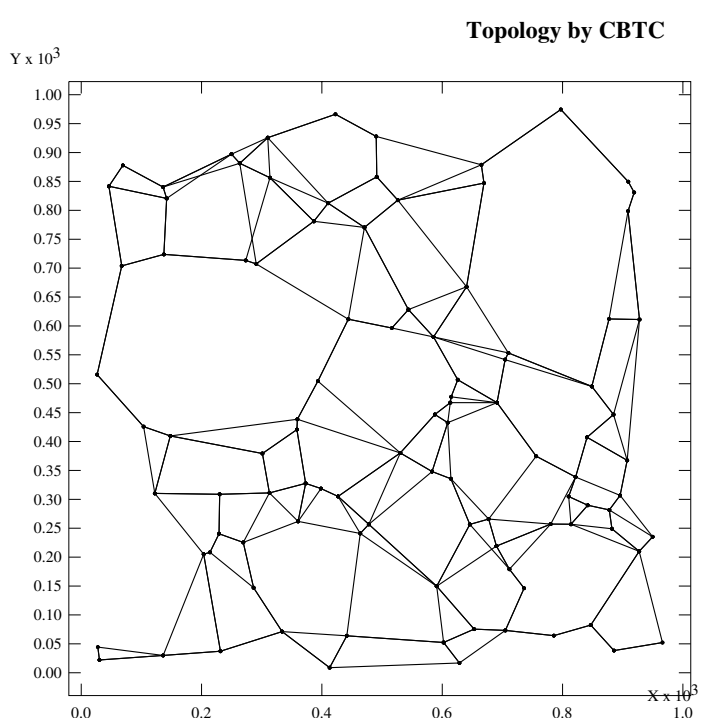

(b) The topology derived under CBTC.

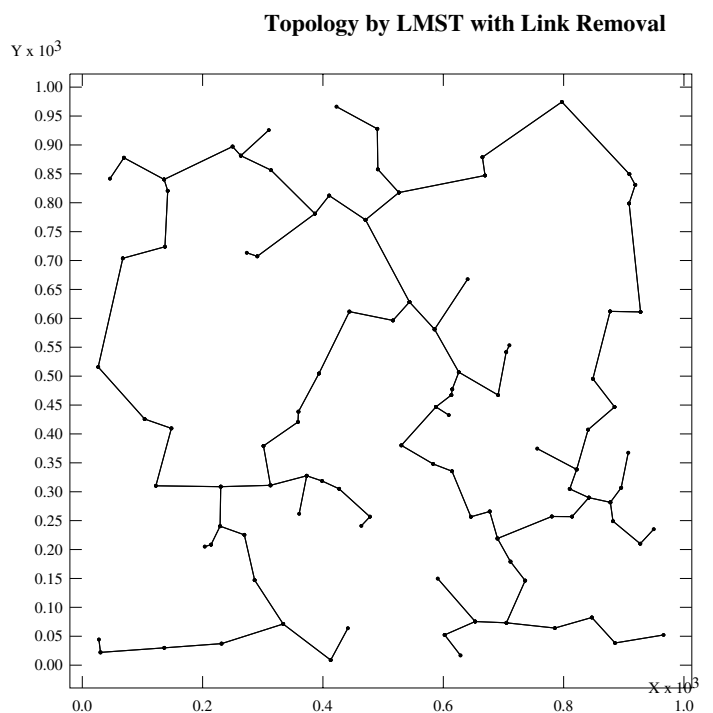

(d) The topology derived under LMST with link removal

Fig. 7. Network topologies derived under different algorithms.

TABLE I

THE MAXIMUM, MINIMUM, AND AVERAGE DEGREES IN THE NETWORK TOPOLOGIES DERIVED USING THE MAXIMUM TRANSMISSION POWER, R\&M(TWO-RAY GROUND MODEL), $C B T C(5 / 6 \pi)$, LMST, AND LMST WITH UNI-DIRECTIONAL LINKS REMOVED.

\begin{tabular}{|c|c|c|c|}
\hline Algorithm & Maximum degree & Minimum degree & Average degree \\
\hline Max trans. power & 28 & 4 & 16.48 \\
\hline CBTC $(5 \pi / 6)$ & 5 & 1 & 2.97 \\
\hline R\&M(Two-ray ground model) & 5 & 1 & 2.64 \\
\hline LMST & 3 & 1 & 2.06 \\
\hline LMST with link removal & 3 & 1 & 2.04 \\
\hline
\end{tabular}




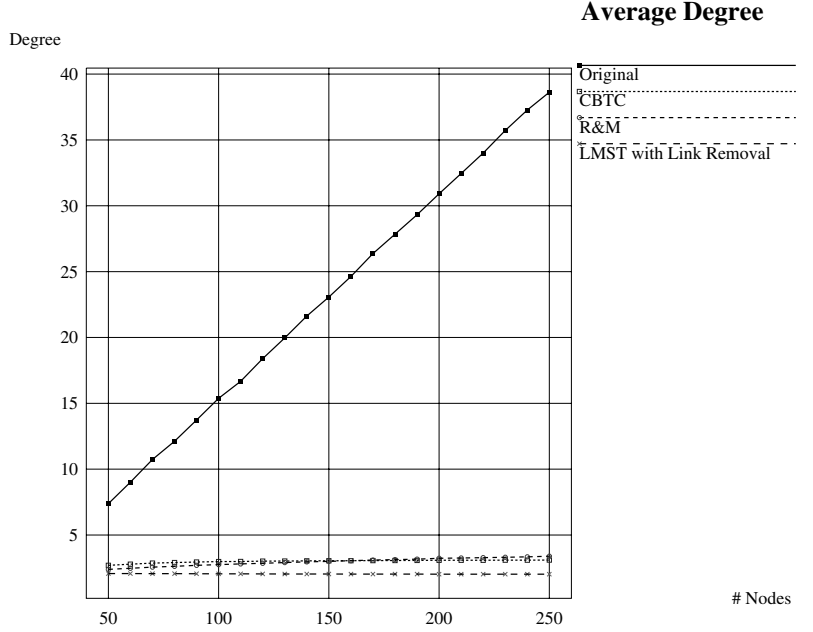

(a) Average degree

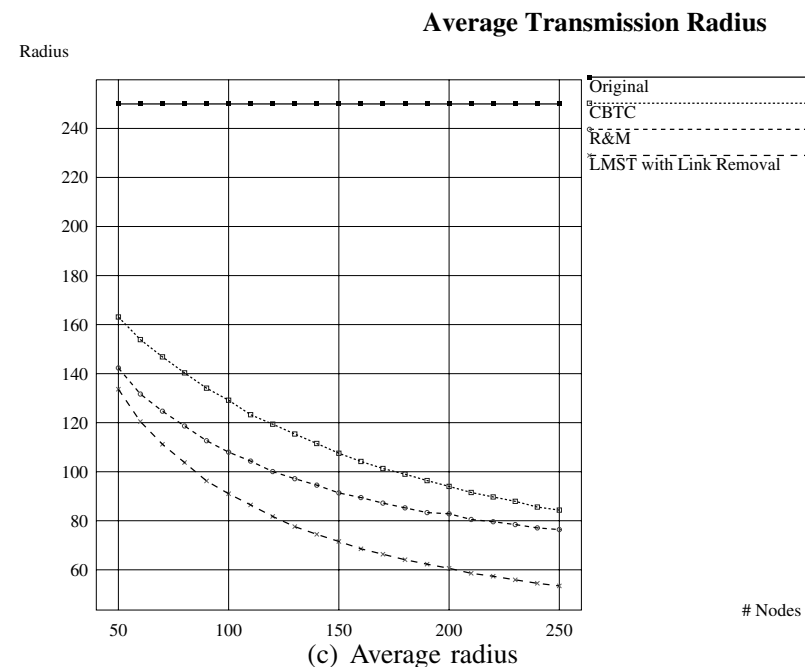

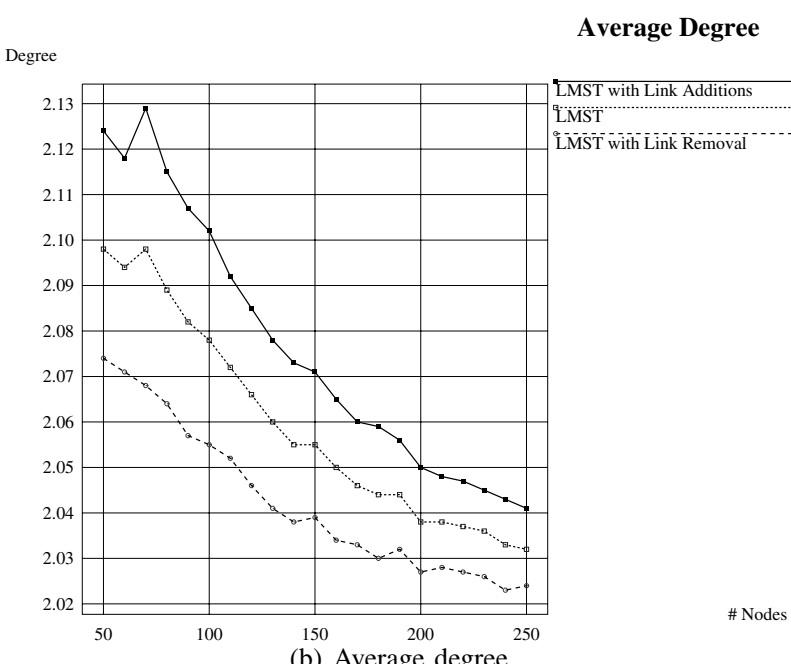

(b) Average degree

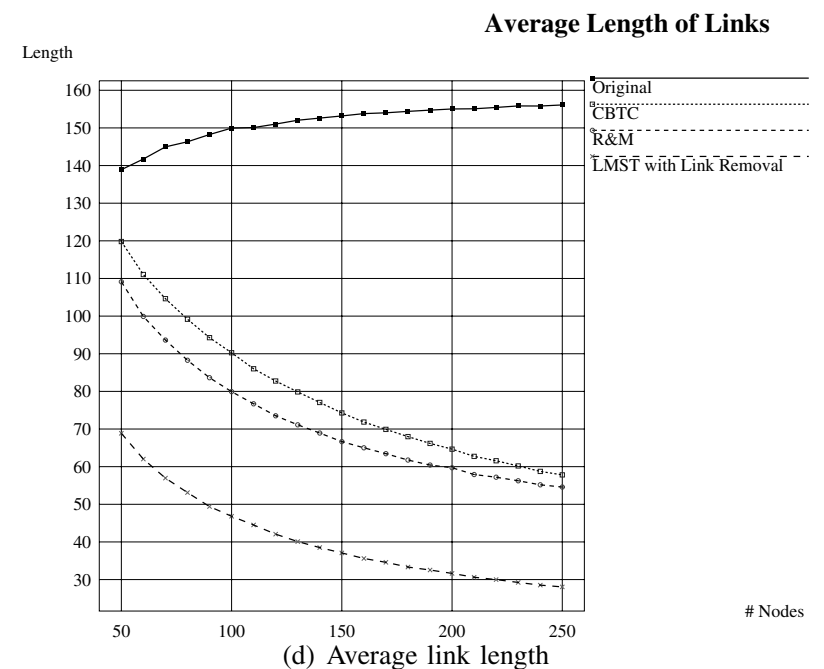

(d) Average link length

Fig. 8. Performance comparisons (w.r.t. degree, radius, and average length of links) among different algorithms.

Two observations are in order: (i) the average node degree under LMST and its two variations does not differ much, and decreases as the node density increases. This is in contrast with the observation that the average node degree of the topology generated using the maximum transmission power increases almost linearly; (ii) the average node degree under LMST is very close to that of a global spanning tree, which is known to have the least average node degree $\left(2-\frac{2}{n} \rightarrow 2\right.$, as $\left.n \rightarrow \infty\right)$ among all the spanning subgraphs. The average radius and the average link length for the topologies generated using the maximum transmission power, R\&M, CBTC, and LMST with link removal are shown, respectively, in Figure 8(c) and 8(d). LMST outperforms in both cases.

In the third simulation, we compare the average power needed to transmit a packet to the sink node located at the center of the region under different propagation channel models. The setting is similar to the previous simulations, and the comparison is made between LMST and R\&M, since the latter has been proved in [9] to find the minimum power topology. As shown in Figure 9, LMST uses slightly more power than R\&M, due to the fact that LMST does not utilize, or optimize its operations with respect to, any information of the propagation channel model.

\section{CONCLUSION}

In this paper, we present a decentralized MST-based topology control algorithm (LMST) for wireless multi-hop networks with limited mobility. As each node builds its local minimum spanning tree independently using locally collected information, the algorithm incurs less message overhead/delay in constructing the topology, and can perform local repair in the case of mobility. The algorithm also has several nice properties: (1) the topology derived preserves the network connectivity; (2) the degree of any node in the topology is bounded by 6 ; and (3) the topology can be transformed into one with bi-directional links (without impairing the network connectivity) after removal of all uni-directional links.

The simulation results show that the topology under LMST 


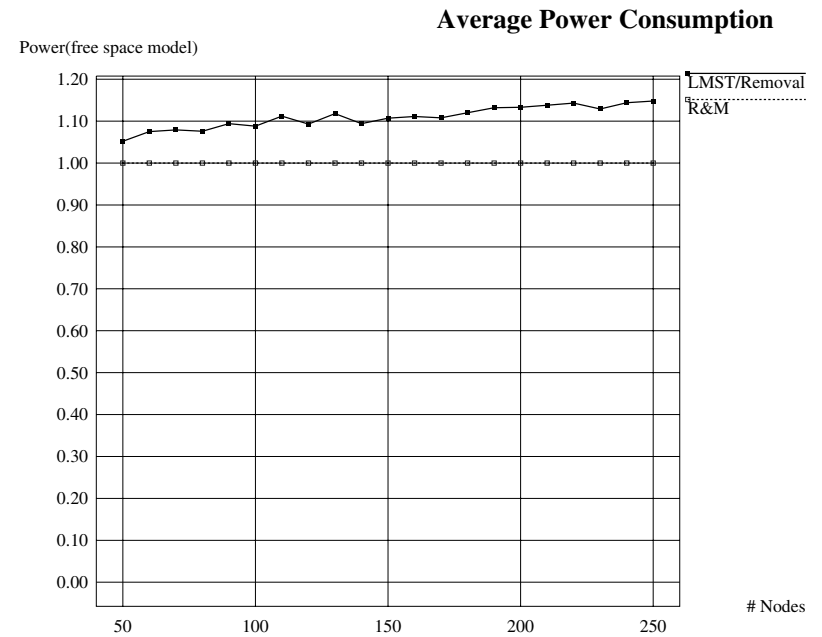

(a) Free space model with $\alpha=4$.

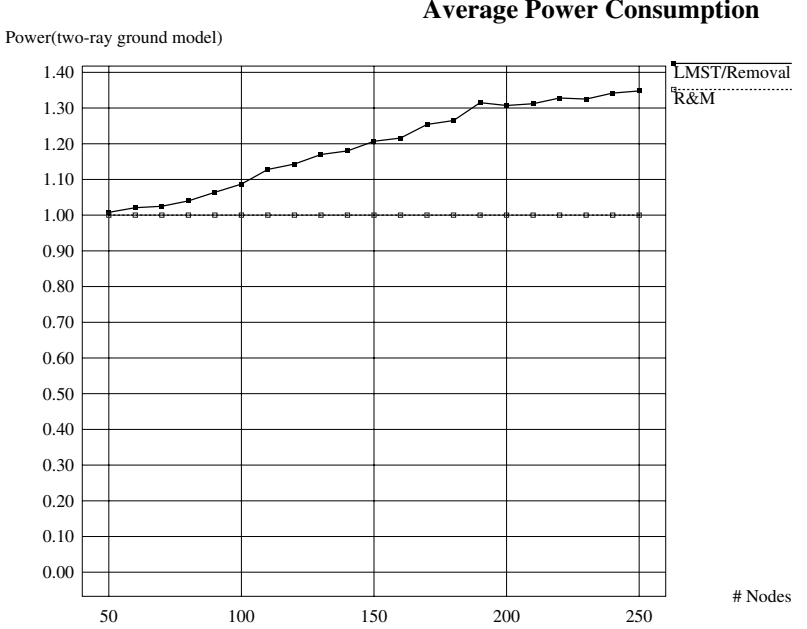

(b) Two-ray ground model.

Fig. 9. Performance comparisons (w.r.t. power consumption) between LMST (with uni-directional link removal) and R\&M.

has a small average node degree (which is very close to the theoretical bound), and a small average radius. The former reduces the MAC-level contention, while the latter implies a small transmission power needed to maintain connectivity.

Our approach tries to minimize the overhead to maintain a connected topology in a dynamic wireless ad hoc network. Therefore, we constructed a minimal topology which approximates a tree structure. However, there is a trade-off between the redundancy and the stability of the topology. Also the topology control algorithm has impact on other layers, especially MAC and routing layer. Those issues will be addressed in our future research.

\section{REFERENCES}

[1] C. E. Jones, K. M. Sivalingam, P. Agrawal, and J. C. Chen, "A survey of energy efficient network protocols for wireless networks," Wireless Networks, vol. 7, no. 4, pp. 343-358, Aug. 2001.

[2] J. Broch, D. A. Maltz, D. B. Johnson, Y.-C. Hu, and J. Jetcheva, "A performance comparison of multi-hop wireless ad hoc network routing protocols," in Proc. ACM/IEEE International Conference on Mobile Computing and Networking (MOBICOM 1998), Dallas, Texas, United States, 1998 , pp. 85-97.

[3] D. A. Maltz, J. Broch, J. Jetcheva, and D. Johnson, "The effects of on-demand behavior in routing protocols for multi-hop wireless ad hoc networks," IEEE J. Select. Areas Commun., vol. 17, no. 8, pp. 14391453, Aug. 1999.

[4] P. Gupta and P. R. Kumar, "The capacity of wireless networks," IEEE Trans. Inform. Theory, vol. 46, no. 2, pp. 388-404, Mar. 2000.

[5] S. Narayanaswamy, V. Kawadia, R. S. Sreenivas, and P. R. Kumar, "Power control in ad-hoc networks: Theory, architecture, algorithm and implementation of the compow protocol," in Proc. of European Wireless 2002, Next Generation Wireless Networks: Technologies, Protocols, Services and Applications, Florence, Italy, Feb. 2002, pp. 156-162.

[6] P. Santi, D. M. Blough, and F. Vainstein, "A probabilistic analysis for the range assignment problem in ad hoc networks," in Proc. ACM Symposium on Mobile Ad Hoc Networking and Computing (MOBIHOC 2001), Long Beach, California, United States, Aug. 2000, pp. 212-220.

[7] L. Li, J. Y. Halpern, P. Bahl, Y.-M. Wang, and R. Wattenhofer, "Analysis of a cone-based distributed topology control algorithm for wireless multi-hop networks," in Proc. ACM Symposium on Principles of Distributed Computing, Newport, Rhode Island, United States, Aug. 2001, pp. 264-273.
[8] R. Ramanathan and R. Rosales-Hain, "Topology control of multihop wireless networks using transmit power adjustment," in Proc. IEEE INFOCOM 2000, Tel Aviv, Israel, Mar. 2000, pp. 404-413.

[9] V. Rodoplu and T. H. Meng, "Minimum energy mobile wireless networks," IEEE J. Select. Areas Commun., vol. 17, no. 8, pp. 1333-1344, Aug. 1999.

[10] E. Bommaiah, M. Liu, A. McAuley, and R. Talpade, "Amroute: Adhoc multicast routing protocol," Aug. 1998, work in progress.

[11] J. J. Garcia-Luna-Aceves and E. L. Madruga, "The core-assisted mesh protocol," IEEE J. Select. Areas Commun., vol. 17, no. 8, pp. 1380-1394, Aug. 1999.

[12] S.-J. Lee, M. Gerla, and C.-C. Chiang, "On-demand multicast routing protocol," in Proc. IEEE Wireless Communications and Networking Conference (WCNC 1999), New Orleans, LA, United States, Sept. 1999, pp. $1298-1302$.

[13] E. M. Royer and C. E. Perkins, "Multicast ad hoc on-demand distance vector (maodv) routing," IETF Internet-Draft, draft-ietf-manet-maodv00.txt, July 2000, work in progress.

[14] C. W. Wu, Y. C. Tay, and C.-K. Toh, "Ad hoc multicast routing protocol utilizing increasing id-numbers (amris)," IETF Internet-Draft, draft-ietfmanet-amris-spec-00.txt, Nov. 1998, work in progress.

[15] S. Basagni, D. Turgut, and S. K. Das, "Mobility-adaptive protocols for managing large ad hoc networks," in Proc. IEEE International Conference on Communications (ICC 2002), Helsinki, Finland, June 2001, pp. 1539-1543.

[16] M. G. J. Wu and I. Stojmenovic, "On calculating power-aware connected dominating sets for efficient routing in ad hoc wireless networks," in Proc. International Conference on Parallel Processing, Vancouver, British Columbia, Canada, Aug. 2002, pp. 346-354.

[17] C. Kee, H. Jun, D. Yun, B. Kim, Y. Kim, B. W. Parkinson, T. Langenstein, S. Pullen, and J. Lee, "Development of indoor navigation system using asynchronous pseudolites," in Proc. 13th International Technical Meeting of the Satellite Division of the Institute of Navigation (ION GPS 2000), Salt Lake City, Utah, United States, Sept. 2000, pp. 1038-1045.

[18] R. Prim, "Shortest connection networks and some generalizations," The Bell System Technical Journal, vol. 36, pp. 1389-1401, 1957.

[19] M. L. Fredman and R. E. Tarjan, "Fibonacci heaps and their uses in improved network optimization algorithms," Journal of the ACM (JACM), vol. 34, no. 3, pp. 596-615, July 1987.

[20] C. Monma and S. Suri, "Transitions in geometric minimum spanning trees," in Proc. ACM Symposium on Computational Geometry, North Conway, New Hampshire, United States, 1991, pp. 239-249.

[21] I. Chlamtac and A. Farago, "Making transmission schedules immune to topology changes in multi-hop packet radio networks," IEEE/ACM Trans. Networking, vol. 2, no. 1, pp. 23-29, Feb. 1994.

[22] J. H. Ju and V. O. K. Li, "An optimal topology-transparent scheduling method in multihop packet radio networks," IEEE/ACM Trans. Networking, vol. 6, no. 3, pp. 298-306, June 1998. 